\title{
La situación actual del deporte universitario en España para el alumnado universitario
}

\section{The current situation of university sport in Spain for university students}

\author{
Pablo Blanco García ${ }^{1 *}$ y Pablo Burillo ${ }^{2}$ \\ 1 Director de Programas Deportivos del Patronato Deportivo Municipal de Gijón \\ 2 Doctor en el Departamento de CAFD de la Universidad Europea de Madrid
}

\begin{abstract}
Resumen: La actividad deportiva ha sufrido un creciente auge en las últimas décadas, convirtiéndose en un importante fenómeno social a nivel mundial. En el ámbito universitario, los Servicios del Deporte se encargan de la gestión de todas las tareas relacionadas con la actividad deportiva dentro de los centros académicos con el objetivo de satisfacer las necesidades de los universitarios.

Esta investigación analiza la situación actual del deporte universitario en España a través de las opiniones de 32 alumnos. El método utilizado es el estudio de caso, perteneciente a la metodología cualitativa, y se ha diseñado una entrevista estructurada formada por un total de 14 preguntas abiertas distribuidas en tres bloques.

Pese a que el modelo deportivo universitario se orienta hacia la participación del alumnado, normalmente los estudiantes practican deporte fuera del centro académico. No obstante, tienen interés en llevar a cabo ejercicio físico en la universidad si mejoraran las condiciones.

Palabras clave: Deporte universitario - Servicios del Deporte - Alumnado universitario
\end{abstract}

Abstract: Sport activity has undergone a growing boom in recent decades, becoming an important social phenomenon worldwide. In the university area, the Sport Services are responsible for the management of all the tasks related to the sport activity within the academic centers in order to satisfy the needs of the university students.

This research analyzes the current situation of university sport in Spain through the opinions of 32 students. The method used is the case study, belonging to the qualitative methodology, and a structured interview was designed,formed by a total of 14 open questions distributed in three blocks. Although the university sports model is oriented towards student participation, normally practice sport outside the academic center. However, they are interested in doing physical exercise at the university if conditions improve.

Keywords: University Sport - Sport Services - University Students.

\section{Introducción}

El deporte universitario ha sido definido por el Consejo Superior de Deportes (CSD) como el realizado por estudiantes universitarios y organizado por los Servicios de Deportes de cada universidad, encargados igualmente de fomentar y facilitar la práctica deportiva.

El crecimiento del deporte en las universidades españolas comenzó en los años veinte del siglo XX a través de la constitución de las asociaciones deportivas que impulsaron la práctica deportiva (CSD, 2005a). La Universidad de Zaragoza fue pionera ya que, en junio de 1922, sus estudiantes fundaron la Sociedad Deportiva Universitaria bajo el ideal del deporte para todos e incluso los profesores del propio centro académico se encargaban de dirigir las actividades deportivas.

La Ley de Reforma Universitaria de 1983 y, posteriormente la Ley Orgánica 4/2007 de Universidades, manifiestan que las universidades deben disponer de los medios necesarios para que los alumnos puedan practicar deporte dentro del propio campus. Esta legislación proporciona la completa facultad a los centros académicos en materia deportiva, es de-

Dirección para correspondencia [Correspodence address]: Pablo Blanco García. E-mail: blancog.pablo@gmail.com cir, reconoce su capacidad para organizar el deporte de forma interna a través de departamentos encargados de la actividad física y el deporte.

De esta forma, la gestión del deporte universitario se origina en los denominados Servicios del Deporte, que Terol (2006) ha definido como entidades que organizan el deporte universitario a partir de la unión de los propios centros. Dichas organizaciones se encargan de la promoción, coordinación y desarrollo de las actividades deportivas dentro de su ámbito de competencia. Además, Martínez (2003) asegura que establecen una serie de objetivos como son coordinar la formación integral del alumno a través de la actividad física y el deporte, mejorar su calidad de vida a través de la prestación de servicios deportivos y el estudio de todo lo relacionado con las actividades físicas y el deporte.

Cada Unidad de Deportes cuenta con una determinada política deportiva y lleva a cabo una serie de acciones encaminadas a cumplir con su modelo. El objetivo final debe ser la configuración un programa deportivo centrado en las necesidades del alumnado universitario. Dichas necesidades consisten en disponer de una oferta deportiva variada, de calidad y económica, contar con una red de instalaciones deportivas en buen estado de mantenimiento y ofrecer unas óptimas condi- 
ciones académicas que les permitan compaginar los estudios con la práctica deportiva.

Algunos autores como Almorza, Yébenes, Bablé, Rivas, Ronquete y Casado (2011) o Martínez (2003) consideran que la actividad deportiva debe ser una iniciativa complementaria a los estudios académicos del alumnado. Estos Departamentos Deportivos deben contribuir a la formación integral de la comunidad universitaria a través de las actividades físicas y el deporte. Por lo tanto, debemos ser conscientes de los grandes beneficios que presenta la práctica deportiva en la vida de los jóvenes universitarios.

Anteriormente, los modelos deportivos se centraban en la competición pero esta tendencia ha ido cambiando ya que, según CSD (2005a), en la actualidad se ha producido un incremento de las actividades deportivas dirigidas. Diferentes investigaciones (Hernández, García \&Oña, 2002; Quesada \& Díez, 1997) han constatado el aumento del deporte para todos ya que una cuarta parte del alumnado practica deporte, cerca de un tercio en el propio centro, y apuestan ampliamente por las competiciones internas y las actividades de práctica libre.

A nivel institucional, los órganos que se encargan de regular la práctica deportiva universitaria en el ámbito nacional son el Consejo Superior de Deportes (CSD) y el Comité Español del Deporte Universitario (CEDU). El CSD tiene las competencias del Estado en materia deportiva y es el encargado de coordinar las actividades deportivas de ámbito nacional junto con las universidades; así como colabora en la promoción de la práctica deportiva universitaria y es responsable de la participación universitaria internacional. En relación a esto, el CEDU nace en 1988 para coordinar estas funciones vinculadas al deporte en los centros académicos.

El deporte universitario, en palabras de París (1996), es el gran desconocido del deporte español ya que existe un movimiento importante con Servicios Deportivos, instalaciones, competiciones, una oferta variada y considerables inversiones económicas; todo ello sin conocimiento fuera del ámbito universitario.

Este estudio tiene como objetivo conocer la situación actual del deporte universitario español teniendo en cuenta las opiniones del alumnado que cursa sus estudios en los centros académicos.

\section{Métodos}

\subsection{Diseño}

Esta investigación descriptiva se basa en la metodología cualitativa que, en palabras de Pérez Serrano (1994), es un proceso activo, sistemático y riguroso de indagación dirigida donde se toman decisiones sobre lo investigable si pertenece al campo objeto de estudio. El estudio de caso es el método elegido que consiste en el estudio de unos casos determinados que permiten interpretar otros similares y proporciona una gran cantidad de información sobre unos pocos sujetos.

Es un método muy flexible, agrupa y permite la comprensión de determinadas prácticas. Thomas y Nelson (2007) aseguran que determina los problemas críticos y amplia los conocimientos en numerosos aspectos relacionados con la educación física, la docencia, el ejercicio, las ciencias del deporte, etc. En este trabajo, la metodología del estudio de caso es interpretativa ya que se basa en el análisis de los datos para clasificar y conceptualizar la información y teorizar sobre el fenómeno.

\subsection{Muestra}

A través del muestreo de propósito, se estableció un criterio de selección basado en los propios objetivos de la investigación donde se priorizaba la calidad de los sujetos sobre el número de estos (Strauss \& Corbin, 2002).La muestra del alumnado está formada por un total de 32 representantes del Consejo de Delegados de los diferentes centros académicos públicos y privados de España. Tres cuartas partes son hombres y la mayoría están matriculados en universidades públicas (Figura 1).

\section{Titularidad de los centro académicos}

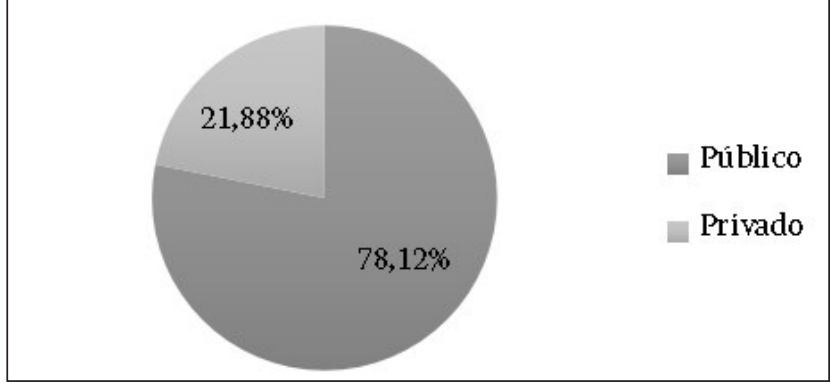

Figura 1. Titularidad de los centros académicos.

Exactamente la mitad de estos sujetos cursan estudios enmarcados en la rama de las Ciencias de la Salud y el resto, de mayor a menor medida, en Ciencias de la Información y Economía, seguido de Derecho, Ingeniería y otras carreras universitarias. Asimismo, más de la mitad del alumnado dispone de algún tipo de formación complementaria, destacando aquellos que cuentan con un postgrado universitario.

Atendiendo a los datos del Ministerio de Educación, Cultura y Deporte, en el curso 2015-2016 un total de 1.548.369 alumnos estaban matriculados en los centros académicos repartidos por el territorio nacional.

\subsection{Instrumentos}

La entrevista estructurada es el instrumento que se ha empleado para conseguir la información de los grupos de infor- 
mantes clave formada por preguntas abiertas y estandarizadas. En un primer lugar, se desarrolló una entrevista piloto previa para el alumnado universitario, que fue revisada y mejorada por expertos, llevándose a cabo también una prueba piloto para adecuar el instrumento a la posterior investigación.

Tanto la validez como la fiabilidad del instrumento se obtuvieron a través de la triangulación de investigadores y consiste, según Fielding y Fielding (1986), en que dos investigadores apliquen la misma entrevista piloto sobre diferentes sujetos y recojan respuestas similares e incluso saturación en las mismas en determinadas preguntas.

\subsection{Variables}

La selección de las variables que constituyen la entrevista estructurada al alumnado se agrupan en tres bloques y, para su configuración, se tuvieron en cuenta diferentes investigaciones vinculadas al deporte universitario que se desarrolla en nuestro país (Almorza et al., 2011; Martínez, 2003; París, 1996; Terol, 2006).

Como se refleja en la siguiente Tabla 1, la entrevista a este grupo de la muestra está formada por 14 preguntas.

Tabla 1. Bloques e ítems de la entrevista.

\begin{tabular}{llc}
\hline Entrevista & Bloques & No de ítems \\
\hline Alumnado universitario & 1- Formación académica & 2 \\
& 2- Práctica deportiva & 4 \\
& 3- Programa deportivo & 8 \\
\hline Total & & 14 \\
\hline
\end{tabular}

\subsection{Procedimiento}

El método utilizado para la obtención de la información empleando una entrevista estructurada estaba constituido por una serie de fases. Se inició contactando con los Servicios de Deportes de los centros académicos nacionales, por vía electrónica, explicando las bases de la investigación y solicitando el contacto de un alumno que actuara como representante del Consejo de Delegados. En caso de respuesta positiva, se procedió a concretar una cita para realizar la entrevista telefónica con el estudiante universitario usando los datos de contacto que nos habían facilitado. Por último, se realizó la mencionada entrevista en la fecha y hora fijada previamente con el alumno.

Se almacenaron todos los datos $y$, por último, se analizaron e interpretaron mediante la utilización del programa Atlas.ti y Excel para Windows.

\section{Resultados}

Los resultados de la entrevista realizada sobre el alumnado universitario se estructuran en tres bloques: Formación académica, Práctica deportiva y Programa deportivo.

\subsection{Formación académica}

A la hora de elegir la universidad donde cursar sus estudios, los alumnos se decantan principalmente por la cercanía al domicilio, seguido del prestigio del centro académico en cuestión. Otras razones minoritarias que argumentan los entrevistados han sido de índole deportivo, la calidad de vida, cuestiones económicas o familiares, de calendario o posibilidades de llevar a cabo investigaciones.

En cuanto al rendimiento académico, la mayoría de estos sujetos están completando sus estudios de forma adecuada, es decir, progresan curso por ańo. El resto de la muestra cuenta con un retraso que puede ser de varias asignaturas e incluso de cursos enteros.

\subsection{Práctica deportiva}

Antes de acceder a la universidad, la práctica totalidad de los entrevistados practicaban actividad física de forma continua$\mathrm{da}$, destacando la participación en competiciones deportivas de modalidades colectivas sobre las individuales y seguido de la inscripción en cursos de diferentes actividades deportivas.

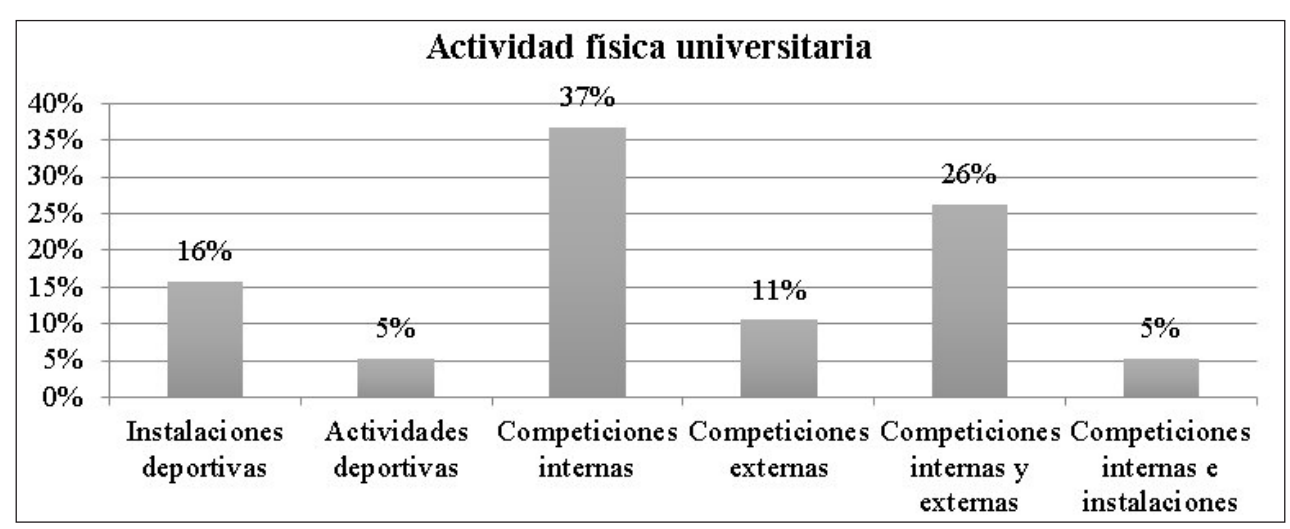

Figura 2. Actividad física universitaria. 
Más de la mitad de los alumnos han tomado parte en la oferta deportiva de su centro académico. Principalmente, participan en competiciones deportivas intrauniversitarias (internas) pero también en competiciones interuniversitarias (externas). Además, se reconoce la utilización libre de instalaciones y, en último lugar, las inscripciones en actividades del programa deportivo (Figura 2). En ocasiones, han tomado parte en varias de las iniciativas que se han citado anteriormente y que componen la oferta deportiva universitaria.

La satisfacción es la tónica habitual de los estudiantes que han participado en alguna actividad del programa deportivo de su universidad; argumentando la buena organización del Servicio de Deportes. También aducen comentarios negativos como la falta de compromiso de los alumnos que se inscriben en las actividades o la problemática de la coincidencia de las clases con entrenamientos o partidos. Mientras que entre las valoraciones negativas, varios de los entrevistados consideran que existe una completa desorganización de la oferta deportiva.

Actualmente, casi todos los entrevistados desarrollan algún tipo de actividad física. Dicha práctica se realiza generalmente fuera del centro académico y se trata, de forma indistinta, de modalidades individuales o colectivas, junto con ejercicio físico de forma libre. Por supuesto, también se localizan sujetos que practican deporte a nivel universitario, sobre todo, a nivel competitivo.

\subsection{Programa deportivo}

Teniendo en cuenta las opiniones del alumnado, el modelo deportivo basado en la participación, también llamado deporte para todos, es el que más abunda en nuestras universidades (Figura 3).

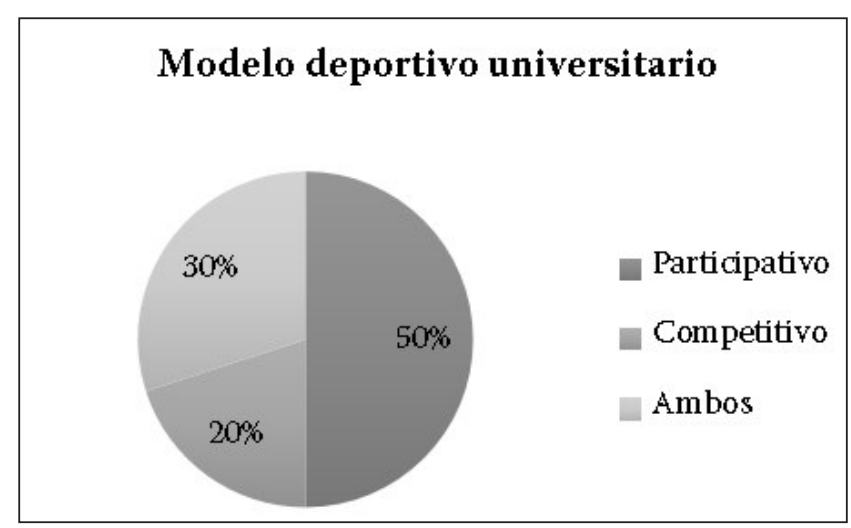

Figura 3. Modelo deportivo universitario.

Se manifiestan opiniones diversas que reconocen la implantación de otros modelos deportivos en los Departamentos Deportivos, tanto centrados en la competición, como una combinación entre este modelo y el participativo.

Muchas universidades disponen de una completa oferta de actividades y cursos que integran su programa deportivo y que está dirigida a sus usuarios. Entre sus principales características, precios reducidos y prioridad para la comunidad universitaria.

En cuanto a las competiciones deportivas que se organizan desde las universidades, una mayoría de los sujetos de la muestra se posiciona de forma favorable. Por lo general, reflejan que existe una buena organización de los partidos para no faltar a clases; así como también aducen otras causas positivas como la elevada participación del alumnado, la posibilidad de conocer a más compańeros o el buen ambiente reinante en dichos eventos. Por otra parte, encontramos sujetos que mantienen pros y contras; junto con otro grupo que desaprueba la organización de estos eventos por su falta de seriedad, poca participación, nivel bajo y corta duración.

Aproximadamente tres cuartas partes de los estudiantes universitarios han hecho o hacen uso de estos espacios deportivos, sobre todo, para la práctica libre y también para la participación en competiciones deportivas. Cabe mencionar que numerosos comentarios valoran el coste asequible que conlleva la reserva de estas instalaciones.

Dichas instalaciones deportivas universitarias presentan una óptima valoración por parte de los entrevistados. Por lo general, manifiestan que pueden practicar deporte en unas instalaciones en notables condiciones $y$, en gran parte, este positivismo es debido a la existencia de convenios de colaboración para el uso de espacios deportivos municipales. Por otro lado, aquellos sujetos que piensan de forma distinta achacan principalmente a la escasez de instalaciones de diferentes disciplinas deportivas y a su antigüedad las causas de su baja estimación.

Casi siete de cada diez estudiantes valoran como satisfactorias las características que integran el programa deportivo de su centro académico (actividades, competiciones e instalaciones deportivas). Consideran que disponen de una oferta amplia pese a que le atribuyen una latente falta de información; junto con otros aspectos minoritarios. Entre las opiniones negativas, por el contrario, se razona que la oferta es escasa, echan en falta más información; así como una mayor cercanía de las instalaciones al lugar de estudio y una mejora de las condiciones de las mismas (Figura 4).

La mayor parte de la muestra considera que sus compañeros también se encuentran satisfechos con el programa deportivo de su centro académico. Argumentan que existe una oferta variada y amplia, disponen de buenas instalaciones y unas condiciones económicas favorables para la práctica deportiva. Aquellos sujetos descontentos esgrimen como inconvenientes la falta de información y la organización de eventos en horarios difícilmente compatibles con el curso académico; así como demandan una mayor variedad de iniciativas deportivas y otros ajustes minoritarios. 


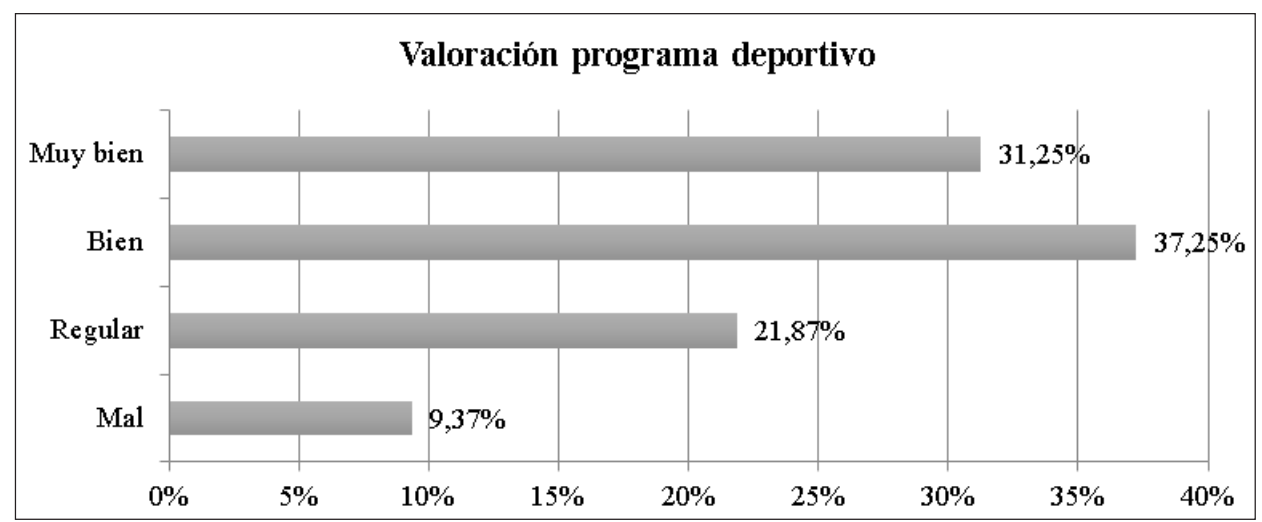

Figura 4. Valoración programa deportivo.

Al preguntarles si llevarían a cabo la práctica deportiva dentro de la universidad si las condiciones fueran las mismas que ofrece un centro deportivo externo, la respuesta más repetida fue afirmativa. El motivo más destacado era la comodidad de realizar ejercicio físico en el propio campus, seguido de estar con los compañeros, un menor coste económico, buenas instalaciones deportivas, el sentimiento de pertenencia o la posibilidad de conseguir créditos de libre elección. En cambio, una minoría prefiere hacer deporte en un centro externo por estar cerca de su domicilio, recibir una atención personalizada o estar con amigos. Adicionalmente, también hay algunos sujetos que optarían por el espacio deportivo que estuviera más cerca de su domicilio sin preferencia alguna.

A continuación, aparece un gráfico que recoge, de forma resumida, los resultados de los diferentes ítems que componen la entrevista al alumnado universitario y los relaciona entre sí (Figura 5).

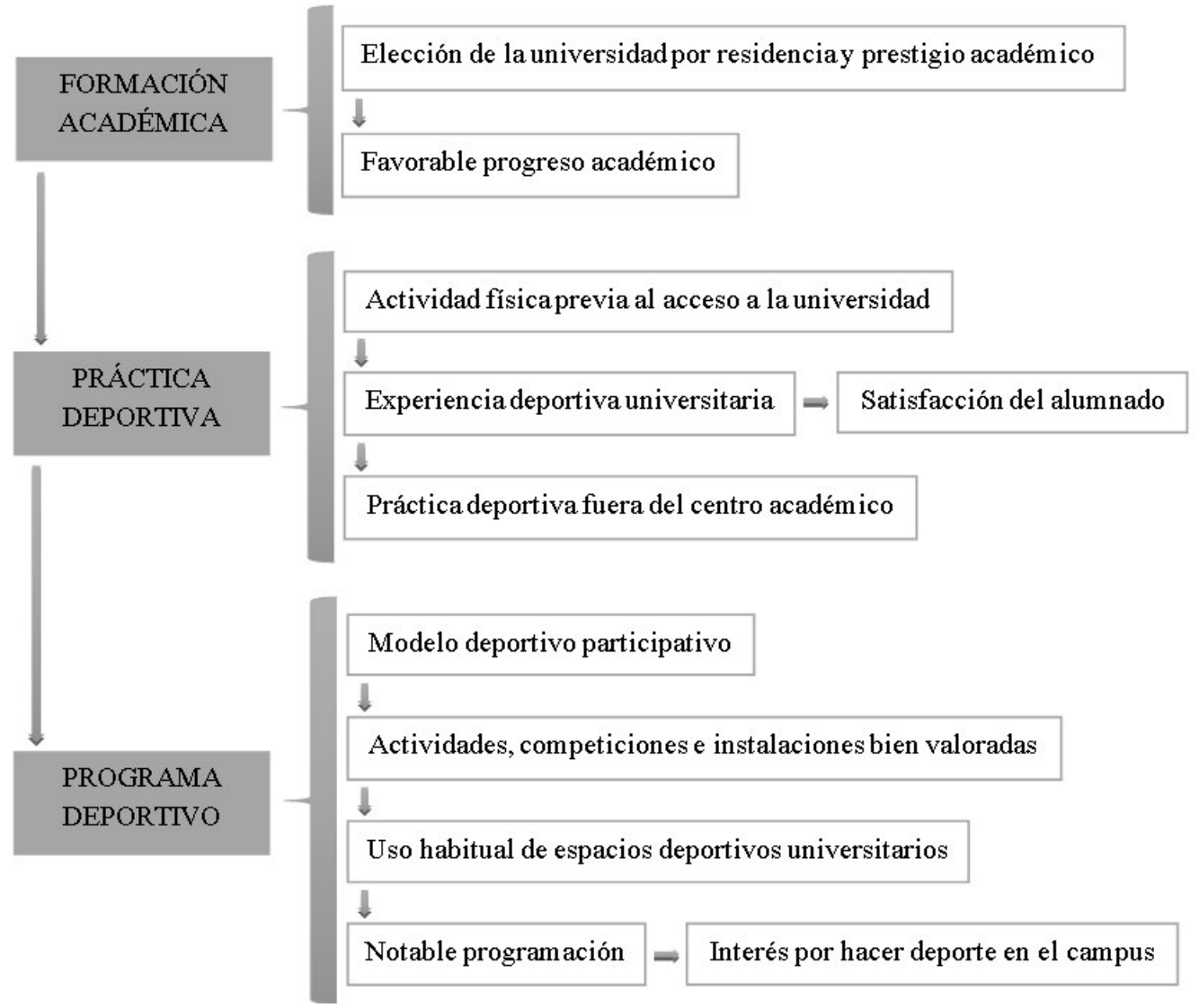

Figura 5. Árbol-estructura de los resultados de la entrevista al alumnado. 


\section{Discusión}

La discusión de los resultados del alumnado universitario se agrupa en dos apartados: Actividad deportiva y Programa deportivo.

\subsection{Actividad deportiva}

La Ley Orgánica de Universidades 4/2007, en el primer punto del artículo 90, refleja que "la práctica deportiva en la universidad es parte de la formación del alumnado y se considera de interés general para todos los miembros de la comunidad universitaria. Corresponde a las universidades en virtud de su autonomía la ordenación y organización de actividades y competiciones deportivas en su ámbito respectivo."

Casi todos los alumnos de este estudio suelen realizar algún tipo de actividad física y, por lo general, dicha práctica se realiza fuera del centro académico. Por lo tanto, resulta alarmante comprobar el elevado porcentaje de alumnos que practican actividades deportivas fuera del centro académico pese a que podrían desarrollarlas dentro de las instalaciones del campus.

En CSD (2005a), con datos más negativos, se manifiesta que el $64 \%$ de los alumnos universitarios practica alguna actividad física, mientras que el $36 \%$ no practica y una parte de ellos (21\%) desearían practicar deporte. Entre los sujetos que practican algún tipo de actividad deportiva, este estudio ha establecido que el $34,6 \%$ la realizan en la universidad, el $63,2 \%$ practican en otra entidad (clubes, instalaciones municipales y centros privados) y, por último, el 2,2\% en ambas organizaciones.

En diferentes investigaciones, París (1996) y Quesada y Díez (1997) han reconocido que en torno a una cuarta parte de los universitarios hacen actividad física en el centro; mientras que en investigaciones más recientes se han hallado datos más negativos ya que Almorza et al. (2011) afirma que no llega a una sexta parte, el $63,43 \%$ son hombres y el $36,57 \%$ mujeres.

Según otros estudios, los alumnos que practican algún tipo de actividad deportiva suponen el $41,9 \%$ de la comunidad universitaria para Castillo y Giménez (2011), el 52,7\% para Pavón (2004), el 53\% para Cecchini y González (2008) y para López (2002) sobre dos tercios.

En nuestra investigación, más de la mitad de los estudiantes han tomado parte o participan en la oferta propuesta por los Servicios Deportivos de su centro académico. Entre ellos, casi cuatro de cada diez han competido internamente, algo más de una carta parte en competiciones internas y externas; así como también, con menores porcentajes, han usado las instalaciones, competido exclusivamente en competiciones interuniversitarias o participado en actividades del programa deportivo.
García Ferrando (2015) ha estipulado que en torno al 75\% del alumnado practica deporte, sobre todo, de forma libre y no competitiva; y aproximadamente el $20 \%$ lo lleva a cabo en el centro académico, tanto en equipos de competición como escuelas, cursos o utilizando las propias instalaciones. Además, únicamente el 15,8\% sobre el total de la población practica deporte en su centro de enseñanza y un $2,4 \%$ en el centro de trabajo.

Almorza, Yébenes, Rivas y Bablé (2008) consideran que el alumnado representa el $45,67 \%$ de los usuarios de una Departamento de Deportes, mientras que el personal es el $6,26 \%$, otros usuarios suponen el $9,01 \%$ y usuarios sin tarjeta deportiva el $39,06 \%$.

Teniendo en cuenta los datos anteriores, las Unidades Deportivas de los centros académicos nacionales deben establecer una política deportiva que estimule la participación del alumnado en su oferta debido a sus innumerables beneficios, tanto fisiológicos como psicológicos. Por ejemplo, a través de un programa que cuente con numerosas actividades deportivas (de sala, acuáticas, etc.), equipos de competición, recompensar la práctica con créditos de libre elección, precios asequibles, torneos, etc.

\subsection{Programa deportivo}

Como recoge el Estatuto del Estudiante, aprobado a través del Real Decreto 1791/2010, en el primer punto del artículo 62, "las actividades deportivas de los estudiantes universitarios podrán orientarse hacia la práctica de deportes y actividades deportivas no competitivas o hacia aquellas organizadas en competiciones internas, autonómicas, nacionales o internacionales".

El grupo de la muestra ha señalado que el deporte participativo, también llamado deporte para todos, es el modelo que prevalece en los Servicios de Deportes de los centros académicos nacionales. Secundado por una combinación entre el deporte competitivo y el participativo y, en último lugar, únicamente la faceta competitiva.

Los modelos deportivos, teniendo en cuenta la investigación del CSD (2005b), se centraban en el deporte de competición pero, en los últimos años, se han ido dirigiendo hacia los programas deactividades deportivas. En este sentido, Hernández, García y Oña (2002) y Quesada y Díez (1997) reconocen que se han producido cambios en las últimas décadas hacia la preponderancia del deporte para todos ya que los universitarios prefieren ampliamente las competiciones internas y las actividades lúdicas, de mantenimiento de la salud, de práctica libre o en el medio natural.

París (1996) ya señalaba la importancia del deporte participativo entre toda la oferta universitaria ya que la práctica se distribuye en competiciones internas el $48 \%$, de promoción y recreación el $42 \%$ y espaciales el 10\%. En referencia al gasto en materia deportiva, se divide en promoción y re- 
creación el $46 \%$ y en competiciones externas $32 \%$ e internas $22 \%$.

El modelo participativo cumple con los verdaderos valores del deporte universitario al perseguir la práctica deportiva de todos los universitarios a través de una variada oferta de actividades deportivas, competiciones internas y la disponibilidad de una completa red de instalaciones. "La finalidad fundamental de un Servicio de Deportes no es hacer campeones, sino complementar la formación de los estudiantes a través de la actividad fisico-deportiva que la nueva realidad social deman$d a$ "(Martínez 2006:181).

Tres cuartas partes de los alumnos del estudio certifican que los Departamentos de Deportes han configurado un programa completo de actividades para sus usuarios. Exactamente una tercera parte de la muestra, según CSD (2005a), no conoce ninguna actividad que desarrolle su universidad. Entre la oferta, el 45\% son de promoción deportiva, seguido de las actividades cuya finalidad es la competición con un $23,57 \%$, las de preparación física con un 14,28\%, en el medio natural con un $11,42 \%$, las actividades especiales con un $4,26 \% \mathrm{y}$, por último, las actividades de expresión con un $1,42 \%$. Además, la prestación de servicios constituye un gasto del 23,18\% sobre el total del presupuesto del deporte universitario y supone dos tercios de los ingresos de los departamentos.

CSD (2009) afirma que las Unidades de Deportes cuentan con unos programas anuales donde ofertan aproximadamente 30 actividades clasificadas en tres ámbitos denominados salud, aprendizaje deportivo y actividades en la naturaleza.

Es totalmente indispensable que un centro académico tenga una completa oferta de actividades deportivas dirigida a todos los usuarios y de diferente tipología (de sala, acuáticas, etc.) bajo la finalidad de potenciar el deporte saludable. El Plan Integral para la Actividad Física y el Deporte del CSD constata que las universidades ofertan como media veinticinco actividades de aprendizaje deportivo y de salud; la cifra más alta de una universidad son 70 y la más baja 2 actividades.

En referencia a las instalaciones deportivas, en palabras de Almorza, et al. (2008) el número de instalaciones varía mucho entre las universidades siendo la media la veintena, con 9.565 usuarios por espacio. Concretamente, el 67\% de los centros tienen entre 0 y 25 instalaciones aunque existen universidades con más de 50 instalaciones. Con mayor ratio de utilización se sitúan los polideportivos cubiertos con una media de 52.919 usuarios, seguido de salas con 38.919 y de pistas polideportivas descubiertas con 26.677 usuarios.

Aproximadamente tres cuartas partes de los estudiantes universitarios han hecho o hacen uso de estos espacios deportivos, sobre todo, destinada a la práctica libre y competiciones deportivas. Asimismo, valoran los espacios deportivos universitarios de forma positiva al reflejar que se encuentran en unas condiciones notables. Por el contrario, otros sujetos han expresado la escasez de instalaciones y su antigüedad como aspectos negativos.

El estudio del CSD (2005a) deja patente que el 29,80\% de los entrevistados no conoce ninguna instalación deportiva en su universidad. Teniendo en cuenta a diferentes autores como Martínez-Tur, Marzo, Gosálvez y García-Buades (1998) o Parejo y Sánchez (2005) en relación al mantenimiento de los espacios deportivos, es muy importante ya que proporciona un servicio de mejor calidad, reduce costes, alarga la vida de la instalación, etc. El grado de conservación de las instalaciones, según París (1996), es normal en el 34\% de los campus, bueno en el $29 \%$, malo en el $21 \%$, excelente un $10 \%$ y muy malo en el $6 \%$ de los centros académicos.

Morales (2009) ha revelado que el 58,44\% de los usuarios valoran las instalaciones como positivas debido a factores como mantenimiento, espacio, accesos, material, iluminación, temperatura y seguridad; siguiendo las opiniones de la muestra del estudio. Además, Aguado (2006) ha determinado que las instalaciones deportivas de las universidades nacionales se encuentran en condiciones de mantenimiento desiguales; así como se sirven de espacios externos a través del establecimiento de convenios con entidades.

Disponer de una completa infraestructura deportiva y, sobre todo, en buen estado, permite a las universidades ofrecer el desarrollo de una amplia gama de actividades deportivas a todos sus usuarios y cumplir con las disposiciones relacionadas con la práctica deportiva recogidas en la Ley Orgánica de Universidades o en el Estatuto del Estudiante Universitario. Para ello, también pueden valerse de convenios con entidades deportivas para el uso de sus espacios deportivos. Peiró, Ramos, González, Rodríguez y Tordera (1995:67) consideran que los centros académicos deben perseguir "la eficacia en el funcionamiento de las instalaciones, considerando tanto la rentabilidad económica como social de las mismas y los intentos de aplicar criterios de profesionalidad y eficacia empresarial en la dirección de las mismas".

Atendiendo a las opiniones de la mayor parte del alumnado universitario, el programa configurado por los Departamentos Deportivos de las universidades ha sido evaluado de forma satisfactoria. Principalmente, esto es debido a que cuenta con una oferta amplia aunque se manifiesta una considerable falta de información.

"Hay servicios deportivos en todas las universidades, hay instalaciones deportivas universitarias, hay ofertas variadas, competiciones en todos los niveles y una gran inversión económica en infraestructura deportiva para las universidades" (Carrión, 2006:105).

En esta misma línea se mantiene Morales (2009) al afirmar que el $69,42 \%$ de los usuarios valoran la oferta de actividades deportivas como positiva, amplia, amena o variada. Según este autor, conocen el Servicio de Deportes de su universidad el $86,6 \%$ de los alumnos que hacen 
deporte y el $80,8 \%$ de los no practicantes. La valoración del trabajo que efectúan dichos departamentos, según el estudio del CSD (2005a), es positiva para el 95,09\% de los sujetos. Concretamente, el 26,01\% de estos puntúa el rendimiento con un seis, el 52,60\% con un ocho, y el 16,76\% con un diez.

Todo programa deportivo de un centro académico nacional dirigido al alumnado debe contar con tres pilares fundamentales: un conjunto de actividades deportivas de diferente tipología, competiciones individuales y colectivas y una red de instalaciones deportivas.

Casi ocho de cada diez alumnos universitarios han reflejado la satisfacción de sus compañeros con el programa deportivo al tener una oferta amplia, buenas instalaciones y condiciones económicas favorables. Por otro lado, se muestran críticos con la falta de información y la organización de eventos en horarios difícilmente compatibles con el curso académico. En base a estos datos, los centros académicos deberían de mejorar en su política de comunicación; así como tratar de organizar las diferentes iniciativas deportivas respetando las clases y períodos de evaluación.

Datos similares se hallan en la investigación de Almorza et al. (2008), donde el 70,59\% de los usuarios deportivos están satisfechos con los Servicios de Deportes. Asimismo, los niveles de satisfacción son del $81,10 \%$ en las actividades deportivas, el $74,19 \%$ en competiciones, personal propio con el $72,47 \%$ y el $72,30 \%$ con instalaciones deportivas.

\section{Conclusiones}

Por último, tras la discusión de los resultados de la investigación, se presentan a continuación una serie de conclusiones acerca de la situación actual del deporte universitario nacional teniendo en cuenta las opiniones del alumnado:

- Formación académica: El estudiante universitario elige el centro académico donde cursar sus estudios basándose, principalmente, en la cercanía al domicilio y en el prestigio académico del mismo. Por lo general, el progreso académico de los estudiantes sigue la planificación curricular.

- Práctica deportiva: La actividad física, bajo cualquiera de sus diferentes manifestaciones, estaba presente en la vida de estos sujetos antes de iniciar la etapa universitaria. También es común que hayan desarrollado algún tipo de práctica deportiva en el propio centro académico con un balance satisfactorio. Sin embargo, la mayoría de ellos realiza deporte fuera del centro académico.

- Programa deportivo: El modelo vigente en los centros académicos persigue la participación deportiva, también llamada deporte para todos; mientras que el programa deportivo es valorado de forma notable por el alumnado al contar con una completa oferta. Dicha oferta está constituida por numerosas actividades deportivas, la organización de competiciones internas y externas y la disponibilidad de una red de instalaciones. Además, a los estudiantes universitarios les gustaría realizar actividad física en la universidad si mejoraran las condiciones en vez de en centros deportivos externos.

\section{Referencias}

1. Almorza, D., Yébenes, A., Rivas, R. \& Bablé, J.A. (2008). Estudio sobre los programas de convalidación de créditos de libre elección por realización de actividades deportivas en las universidades españoles. Cádiz: Universidad de Cádiz.

2. Almorza, D., Yébenes, A., Bablé, J.A., Rivas, R., Ronquete, J. \&Casado, I. (2011). Estudio Diagnóstico Deporte Universitario Español. Cádiz: Universidad de Cádiz

3. Aguado, J.L. (2006). Estructura del deporte universitario. En Terol, R. (2006), El deporte universitario en España: Actualidad y perspectivas de futuro. Madrid: Dykinson.

4. Carrión, C. (2006). La mujer universitaria y el consumo de actividad físico-deportiva. Valencia: Universidad de Valencia.

5. Castillo, E. \& Giménez, F.J. (2011). Hábitos de práctica de actividad física del alumnado de la Universidad de Huelva. Revista Internacional de Medicina y Ciencias de la Actividad Física y del Deporte, 11 (41), 145163.

6. Cecchini, J.A. \& González, C. (2008).Motivos por los que los estudiantes universitarios no practican deporte. Revista Fuentes, 8, 199 208.

7. Consejo Superior de Deportes (2005a). El modelo deportivo universitario español. Madrid: Ministerio de Educación, Cultura y Deporte. Consejo Superior de Deportes.

8. Consejo Superior de Deportes (2005b). Estudio sobre los modelos de de- porte universitario en Europa. Madrid: Ministerio de Educación, Cultura y Deporte. Consejo Superior de Deportes.

9. Consejo Superior de Deportes (2009). Plan integral para la actividad fisica y el deporte: Deporte en la Universidad. Madrid: Ministerio de Educación, Cultura y Deporte. Consejo Superior de Deportes.

10. Fielding, N.G.,\& Fielding, J.L. (1986). Linking data. Beverly Hills, California: Sage.

11. García Ferrando, M. (2015). Encuesta sobre hábitos deportivos de los españoles. Madrid: Ministerio de Educación, Cultura y Deporte. Consejo Superior de Deportes.

12. Hernández, A.I., García, M.E. \& Ońa, A. (2002). Demanda y práctica de actividades físico-deportivas de tiempo libre entre la comunidad universitaria almeriense. Revista Motricidad, 8, 111-139.

13. Ley Orgánica 11/1983, de 25 de agosto, de Reforma Universitaria.

14. Ley Orgánica 4/2007, de 12 de abril, por la que se modifica la Ley Orgánica 6/2001, de 21 de diciembre, de Universidades.

15. López Yeste, A. (2002). Gestión del Deporte Universitario. Actas del I Congreso de Gestión del Deporte de la Asociación de Gestores profesionales de la Comunidad Valenciana (pp. 37-45). Valencia: Asociación de Gestores Profesionales de la Comunidad Valenciana.

16. Martínez, V. (2003). Estructura organizativa de los Servicios de Deporte universitario en España. Madrid: Ministerio de Educación, Cultura y Deporte. Consejo Superior de Deportes. 
17. Martínez, V. (2006). Orientación y tendencias del deporte universitario en España. En Terol, R. (2006), El deporte universitario en España: Actualidad y perspectivas de futuro. Madrid: Dykinson.

18. Martínez-Tur, V., Marzo, J.C., Gosálvez, I. \& García-Buades, E. (1998). El nivel de saturación de las instalaciones deportivas como atributo situacional y variable de la calidad: sus relaciones con la satisfacción de los usuarios. Revista de Psicología del Deporte, 7 (13), 135-146.

19. Morales, M.A. (2009). La organización y gestión de las actividades físico deportivas en la universidad: un caso práctico. Málaga: Universidad de Málaga.

20. Parejo, E. \& Sánchez, E. (2005). El mantenimiento aplicado a las instalaciones deportivas. Barcelona: INDE.

21. París, F. (1996). El deporte en las universidades españolas. Análisis de la encuesta sobre su organización, práctica y equipamientos. Madrid: Ministerio de Educación y Cultura. Consejo Superior de Deportes.

22. Pavón, A.I. (2004). Motivaciones e intereses de los universitarios murcianos hacia la práctica físico-deportiva. Murcia: Universidad de Murcia.

23. Peiró, J.M., Ramos, J., González, P., Rodríguez, I. \& Tordera, N.
(1995). Aspectos psicosociales de la gestión de instalaciones deportivas: Implicaciones sobre las actitudes y conductas deportivas de los usuarios. Apunts: Educación Fisica y Deportes, 39, 67-75.

24. Pérez Serrano, G. (1994). Investigación cualitativa. Retos e interrogantes I: Métodos. Madrid: La Muralla.

25. Quesada, S. \& Díez, M.D. (1997). Convergencia del deporte universitario en Europa. Alicante: Departamento de Economía Aplicada y Política Económica. Universidad de Alicante.

26. Real Decreto $1791 / 2010$, de 30 de diciembre, por el que se aprueba el Estatuto del Estudiante Universitario.

27. Strauss, A., \& Corbin, J. (2002). Bases de la investigación cualitativa. Técnicas y procedimientos para desarrollar la teoría fundamentada. Medellín, Colombia: Universidad de Antioquía.

28. Terol, R. (2006). El deporte universitario en España: actualidad y perspectivas de futuro. Madrid: Dykinson.

29. Thomas, J.R. \& Nelson, J.K. (2007). Métodos de investigación en actividad física. Barcelona: Paidotribo. 